\title{
X-Linked Dominant Congenital Ptosis Cosegregating with an Interstitial Insertion of a Chromosome 1p21.3 Fragment into a Quasipalindromic Sequence in Xq27.1
}

\author{
David J. Bunyan 1,2, David O. Robinson 1,2, Anthony G. Tyers'3, Shuwen Huang4, \\ Vivienne K. Maloney ${ }^{1}$, Francis H. Grand ${ }^{2}$, Sarah Ennis ${ }^{5}$, Samantha R. de Silva6, \\ John A. Crolla1,2, Tristan F. W. McMullan7 \\ ${ }^{1}$ Wessex Regional Genetics Laboratory, Salisbury District Hospital, Salisbury, UK \\ ${ }^{2}$ Human Genetics Division, Southampton University School of Medicine, Southampton, UK \\ ${ }^{3}$ Ophthalmology Department, Salisbury District Hospital, Salisbury, UK \\ ${ }^{4}$ Manchester Centre for Genomic Medicine, Saint Mary's Hospital, Manchester, UK \\ ${ }^{5}$ Genetic Epidemiology and Genomic Informatics Group, Faculty of Medicine, University of Southampton, \\ Southampton, UK \\ ${ }^{6}$ Oxford Eye Hospital, John Radcliffe Hospital, Oxford, UK \\ ${ }^{7}$ Ophthalmology Department, Northampton General Hospital, Northampton, UK \\ Email: Dave.Bunyan@salisbury.nhs.uk
}

Received 20 August 2014; revised 19 September 2014; accepted 18 October 2014

Copyright (C) 2014 by authors and Scientific Research Publishing Inc.

This work is licensed under the Creative Commons Attribution International License (CC BY).

http://creativecommons.org/licenses/by/4.0/

c) (i) Open Access

\section{Abstract}

Blepharoptosis (ptosis) is defined as the abnormal drooping of the upper eyelid and is a feature of many conditions. It can be in isolated or syndromic form, bilateral or unilateral and congenital or acquired. Previously we have carried out linkage analysis on a family with dominantly inherited congenital bilateral isolated ptosis and found the condition to be linked to a region of approximately 20 megabases of chromosome Xq24-Xq27.1 with a cumulative LOD score of 5.89. We now describe further analysis using array comparative genomic hybridisation (array CGH), fluorescence in situ hybridisation (FISH), long range PCR and sequencing. This has enabled us to identify and characterise at the level of DNA sequence an insertional duplication and rearrangement involving chromosomes 1p21.3 and a small quasipalindromic sequence in Xq27.1, disruption of which has been associated with other phenotypes but which is cosegregating with $X$-linked congenital bilateral isolated ptosis in this family. This work highlights the significance of the small quasipalindromic sequence in genomic rearrangements involving Xq27.1 and the importance of comprehensive molecular and molecular cytogenetic investigations to fully characterise genomic struc- 
tural complexity.

Keywords

Ptosis, X-Linked Dominant, Insertional Duplication

\section{Introduction}

Blepharoptosis, usually abbreviated to the term ptosis, is defined as the abnormal drooping of the upper eyelid. It can be bilateral or unilateral, congenital or acquired, in isolated or syndromic form and is a feature of many conditions. When present from birth it can impair the development of full visual acuity and is usually corrected by surgery. Congenital ptosis is caused by underdevelopment of the levator palpebrae superioris muscle which raises the eyelid. The underlying defect is unknown and may be myogenic or neurogenic. The condition can be familial and we have previously carried out linkage analysis on a UK pedigree with dominantly inherited congenital bilateral isolated ptosis which indicated that the causative locus lay within a critical region of approximately $20 \mathrm{Mb}$ of Xq24-27.1 which has since been refined to a region of $18 \mathrm{Mb}$ [1]. The only other documented familial isolated ptosis case is from North America and maps to chromosome 1p32-34.1 [2]. X-linked ptosis has been seen in other families as part of a much broader phenotype which also included microcephaly and digital defects [3], but the candidate genes proposed in those families, FAM45B and ENOX2, are $6 \mathrm{Mb}$ outside of our linkage region.

Because of the limitations of linkage analysis and the number of family members available we were unable to further refine the critical region in our family using this methodology. We now describe a different approach enabling detailed characterisation of a chromosomal rearrangement cosegregating with X-linked ptosis in this pedigree. Array competitive genome hybridisation (aCGH) identified the duplication of a fragment of chromosome 1p21.3 and fluorescence in situ hybridization (FISH) analysis showed the fragment to be inserted into Xq27.1. Likely areas for the insertion breakpoints were then identified by conducting an in silico search for 1) repetitive sequences common to both the critical region of the $\mathrm{X}$ chromosome as identified by FISH and the ends of the inserted fragment as identified by aCGH and 2) potential fragile sites. Long-range PCR across candidate loci was then performed, failure of amplification in patient DNA compared with controls indicating that the insertion lay between specific primers. Subsequent PCR was then carried out using the same X chromosome primers paired with primers homologous to the ends of the inserted chromosome 1 sequence to generate a PCR product spanning the breakpoint. DNA sequencing was then performed to identify the exact location of the insertion in Xq27.1, involving a palindromic sequence previously identified as a site of interchromosomal insertions associated with other phenotypes [4] [5].

In order to exclude the presence of another causative mutation within the $18 \mathrm{Mb}$ critical region of the $\mathrm{X}$ chromosome derived from linkage data, exome sequencing was also performed.

\section{Methods}

\subsection{Patients}

Affected family members exhibit dominantly inherited congenital isolated bilateral ptosis. There is no evidence of epicanthus inversus, blepharophimosis or prevailing ocular motility disorder. Ptosis is symmetrical and equal in both males and females and results in an abnormally low upper eyelid position that impinges upon the visual axis in the primary position of gaze. There is minimal levator function bilaterally, absence of the upper lid skin crease, pronounced frontalis overaction, a characteristic chin-up head posture and no evidence of progression or variability of ptosis. The family pedigree is shown in Figure 1. Consent for diagnostic testing was obtained from all patients as part of the referral process.

\subsection{Array CGH Analysis}

Array CGH analysis was performed initially using a 244 k Agilent array (reference 014693, Agilent Technologies, Santa Clara, CA) followed by an $8 \times 60$ k custom array (Agilent reference 024453) built on an Agilent 


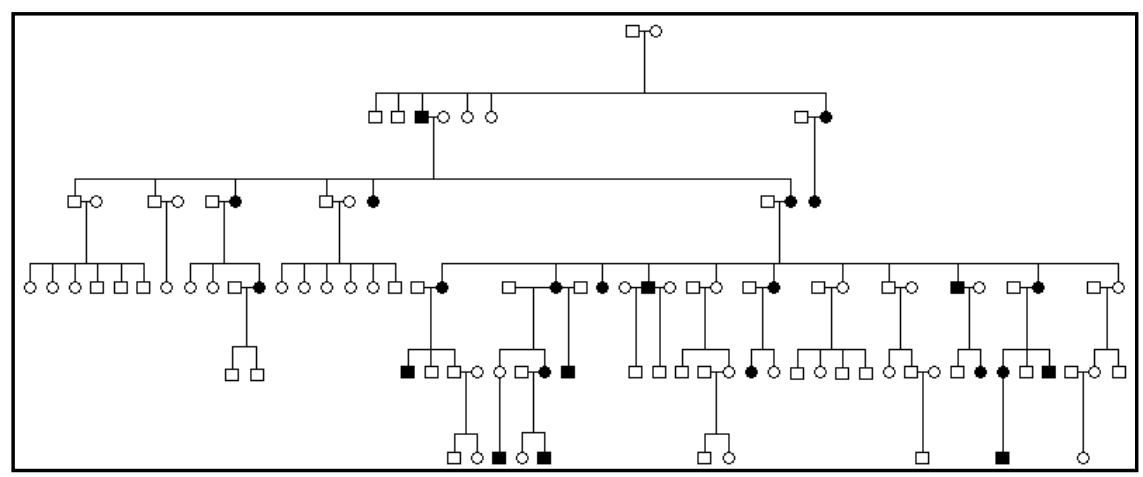

Figure 1. X-linked isolated congenital bilateral ptosis: family pedigree. Black symbols represent affected individuals.

$4 \times 44 \mathrm{k}$ backbone with coverage of approximately 1 probe per 100 base pairs of the approximately $120 \mathrm{~kb}$ area of chromosome 1p21.3 shown to be duplicated by the $244 \mathrm{k}$ array.

\subsection{FISH}

FISH was performed using fluorescence labelled bacterial artificial chromosome clones (BACs) and a plasmid artificial chromosome clone (PAC) from the $30 \mathrm{k}$ human tiling path clone set (ensembl.org/homo_sapiens/index) and standard protocols. A duplication of part of the chromosome 1 DPYD gene (dihydropyrimidine dehydrogenase, MIM 612779), identified by array CGH was verified using the 1p21.3 specific BAC clone RP11-359C24. Further analysis using RP11-359D24 together with Xq27 specific probes BAC RP11-197K18 and PAC RP1177G6 refined the location of the duplicated fragment.

\subsection{Multiplex Ligation-Dependant Probe Amplification (MLPA)}

Dosage analysis was carried out using MLPA [6]. A custom-made MLPA probe was designed specifically for the duplicated chromosome 1 fragment (probe sequence available upon request). $0.5 \mu \mathrm{l}$ of probe at a concentration of $4 \mathrm{fmol} / \mu \mathrm{l}$ was used in conjunction with a commercially available MLPA probe mix (P200, MRC Holland) to provide controls. MLPA was carried out according to the manufacturer's protocol.

\subsection{In Silico Analysis to Define Putative X Chromosome Breakpoints}

Likely sites of insertion of the DPYD fragment in the $\mathrm{X}$ chromosome were identified by an in silico search for homologous sequences common to the critical region of chromosome $\mathrm{Xq}$ (as defined by FISH) and the duplicated fragment of the DPYD gene near the breakpoint areas as defined by array CGH. $1 \mathrm{~kb}$ of sequence at either end of the duplicated fragment was analysed using the BLAT sequence alignment program (genome. ucsc.edu/cgi-bin/hgBlat) to search for homologous sequences in the $\mathrm{X}$ chromosome critical region. A second in silico analysis involved a search for AT dinucleotide rich regions (commonly associated with fragile sites) within the $\mathrm{X}$ chromosome critical region. The $\mathrm{X}$ chromosome sequence was downloaded from Ensembl (ensembl.org) as a text file and a Microsoft Word search function was used to identify AT dinucleotide rich regions within the critical region.

\subsection{Long Range PCR}

Long range PCR was carried out using the "Expand long template system" and buffer 2 (Roche) according to the manufacturer's protocol. Primers were designed within the $\mathrm{X}$ chromosome critical region to give a product of approximately $7.5 \mathrm{~kb}$ in a normal control individual (primer sequences available upon request). PCR was then performed using normal male controls and males with the partial DPYD gene insertion with the premise that the insertion would render the PCR fragment too large to amplify in affected males but not in normal controls. The long range PCR was focused on areas of the critical region of the X chromosome highlighted by in silico analysis. 


\subsection{Identification of Breakpoints}

Following the identification by long range PCR of the approximate site of insertion of the DPYD gene fragment within a $7.5 \mathrm{~kb}$ fragment of the X chromosome, PCR amplification of genomic DNA was performed using combinations of primers from the ends of the inserted DPYD fragment and the relevant long range PCR primers on the $\mathrm{X}$ chromosome (primer sequences available on request) to generate a fragment spanning the insertion breakpoint. Reactions were in a $25 \mu \mathrm{l}$ volume containing 50 ng DNA, $1 \mathrm{mM}$ forward primer, $1 \mathrm{mM}$ reverse primer, $2.5 \mu \mathrm{l} 10 \times$ reaction buffer (Perkin Elmer), $0.16 \mathrm{mM}$ dNTPs, $1.5 \mathrm{mM} \mathrm{MgCl}_{2}, 0.5 \mathrm{u}$ Taq polymerase (Taq Gold, Perkin Elmer). Cycling parameters were $94^{\circ} \mathrm{C}$ for 12 minutes followed by 35 cycles of $94^{\circ} \mathrm{C}$ for 30 seconds, $60^{\circ} \mathrm{C}$ for 30 seconds and $72^{\circ} \mathrm{C}$ for 30 seconds. PCR products were sequenced bi-directionally using the Big Dye ${ }^{\mathrm{TM}}$ Terminator v1.1 cycle sequencing kit (Applied Biosystems, Warrington, UK) using the same primers as for PCR amplification. Results were analysed with Mutation Surveyor version 3.2 (Soft Genetics, State College, PA).

\subsection{Exome Sequencing}

Exome sequencing was carried out on two affected second-cousins from generation VI of the pedigree. Exome capture and sequencing were conducted at the Wellcome Trust Centre for Human Genetics at Oxford University. Data analysis was performed by using an in-house software pipeline, consisting of Novoalign (Novocraft Technologies, Selangor, Malaysia) for read alignment, Samtools [7] for variant calling and Annovar [8] for variant annotation. The detected genetic variants were filtered to reduce the list to those that were non-synonymous, previously unreported (or flagged as clinically relevant) and present in all affected individuals.

\section{Results}

\subsection{Array CGH Analysis}

Array CGH analysis of an affected female member of the pedigree with the Agilent $244 \mathrm{k}$ array detected a duplication of 13 probes on chromosome 1p21.3. Further array CGH analysis on six affected and two unaffected family members using the $8 \times 60 \mathrm{k}$ custom array, which included dense coverage of the duplicated area of chromosome 1, showed 1163 probes to be duplicated and indicated the duplication to be a maximum of bases 1: 97,886,245-98,006,232 and a minimum of bases 1: 97,886,322-98,006,161. The duplication was present in all six affected individuals tested and absent in two unaffected individuals (Figure 2(a)). The duplication was approximately $120 \mathrm{~kb}$ in size and consisted of part of the dihydropyrimidine dehydrogenase (DPYD; MIM 612779) gene with breakpoints in introns 12 and 14. All genomic base numbering is taken from the February 2009 human genome reference sequence GRCh37 (ensembl.org).

\subsection{FISH}

FISH analysis using chromosome 1 BAC probe RP11-359C24, which contains the duplicated fragment, showed the duplicated segment of chromosome 1 to be inserted into chromosome Xq27.1 (Figure 2(b)), i.e. der (X) dir ins (X; 1) (q27.1; p21.3). Further FISH analysis using chromosome 1p21.3 specific BACRP11-359C24 together with X chromosome BAC probes RP11-197K18 (encompassing bases X: 138,767,398-138,827,453) and RP1-177G6 (encompassing bases X: 139,812,182-139,939,308) located the position of the insertion to an area of approximately $1 \mathrm{Mb}$ of DNA in Xq27.1 between these two loci.

\subsection{MLPA}

MLPA analysis confirmed the presence of the duplication in five affected family members and its absence in five unaffected family members (data not shown). It was also shown to be present in two unaffected carrier females previously shown by linkage analysis to have the "at risk" Xq haplotype (see Section 4).

\subsection{In Silico Analysis to Define Putative X Chromosome Breakpoints}

BLAT analysis (genome.ucsc.edu/cgi-bin/hgBlat) to look for homologous DPYD gene fragment sequences failed to find any significant matches within the $\mathrm{X}$ chromosome critical region. However the search for an 


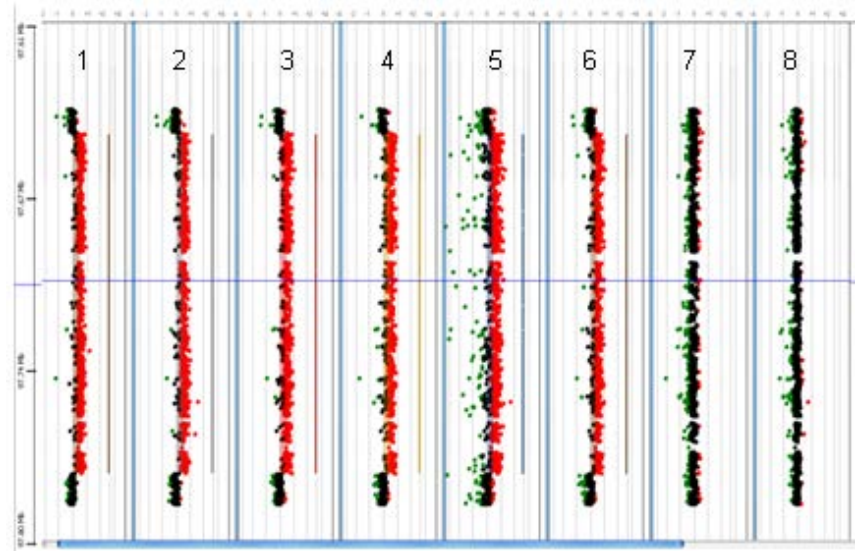

(a)

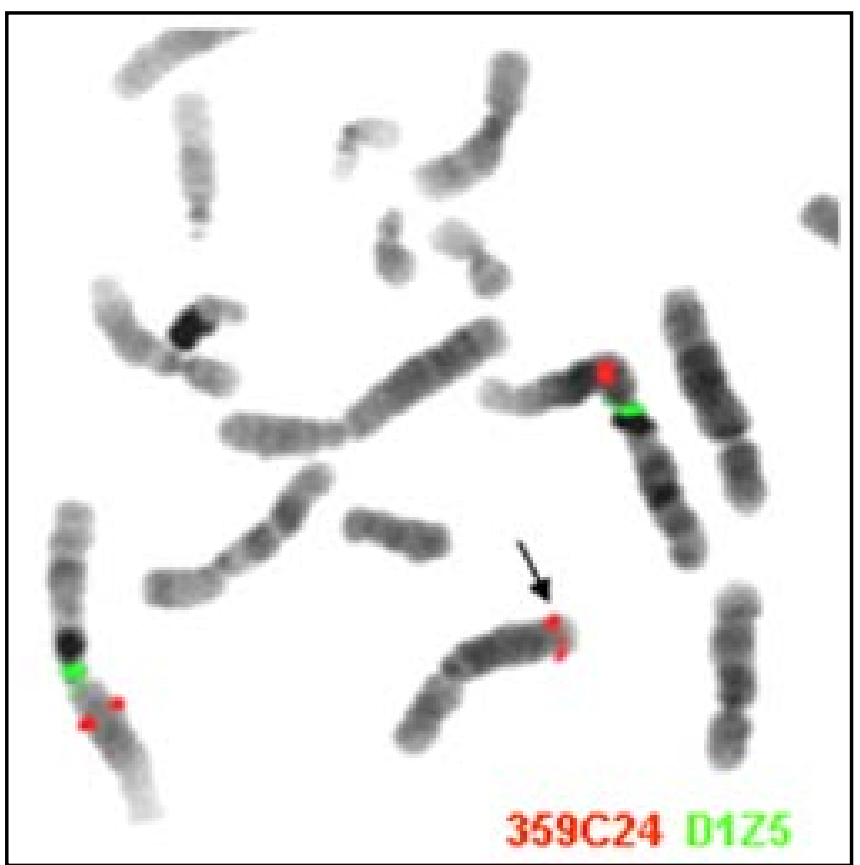

(b)

Figure 2. Identification and location of the duplicated fragment. (a) Array CGH analysis of 6 affected members of the ptosis pedigree (panels 1-6) and 2 unaffected members (panels 7 and 8). Each dot represents an array CGH probe within the chromosome 1p21 region between bases 97,618,814 and 97,802,323. Black dots indicate normal copy number, red dots indicate an additional copy. (b) FISH analysis of an affected member of the ptosis pedigree. Green signal represents probe D1Z5 specific for the chromosome 1 centromere. Red signal represents probe RP11-359C24 specific to the duplicated chromosome. 1p21.3 fragment. The additional copy is inserted into chromosome Xq27 (arrowed).

AT-rich region identified an area of the $\mathrm{X}$ chromosome spanning approximately $2 \mathrm{~kb}$ of sequence (X: 139,493,405-139,495,484) within the $1 \mathrm{Mb}$ critical region defined by FISH.

\subsection{PCR Identification of Breakpoints}

PCR amplification was performed with a series of $\mathrm{X}$ chromosome specific primer pairs in the region of the $\mathrm{X}$ 
chromosome containing the AT-rich region identified above. One primer pair (covering fragment X: $139,498,501-139,506,034)$ gave a PCR product of the expected size with control DNA but no product with DNA from affected males (Figure 3(a)) indicating the possibility that the insertion lay between these two primers. Further PCR analysis using primers homologous to the inserted chromosome 1 fragment paired with X specific primers was then carried out. One primer pair (hybridising to 1: 98,006,082-98,006,100 and X: 139,506,014-139,506,034) generated a PCR product with DNA from affected individuals but not with control DNA (Figure 3(b)) indicating that the $\mathrm{X}$ chromosome breakpoint was within this fragment. Sequence analysis confirmed the presence of both $\mathrm{X}$ chromosome sequence and chromosome 1 sequence and identified an $\mathrm{X}$ chromosome breakpoint at base 139,505,833, approximately $7.5 \mathrm{~kb}$ distal to the AT-rich region (Figure 4(a)).

Subsequent PCR analysis to identify the second X: 1 junction point was unable to generate a product using a primer located close to the distal end of the inserted chromosome 1 sequence paired with an $\mathrm{X}$ primer immediately distal to the $\mathrm{X}$ breakpoint at base $139,505,833$. This suggested a more complex rearrangement than a simple insertion. PCR amplification with the insert specific primer paired with a range of X chromosome primers more distal to the breakpoint region was performed and the second chromosome $\mathrm{X}$ breakpoint was identified at base 139,502,960 which was within a 180 bp quasipalindromic sequence (Figure 4(b)). The DPYD gene fragment insertion was shown to be 119,902 bp in size (bases 1: 97,886,267-98,006,168) which includes the whole of exons 13 and 14, the whole of intron 13 and part of introns 12 and 14.

Further sequence analysis revealed additional complexity of the X chromosome rearrangement. A 427 base pair fragment (X: 139,505,833-139,506,259) has been duplicated, one copy of which is situated immediately distal to the inserted chromosome 1 segment. Also a 7 base pair sequence (X: 139,502,954-139,502,960) has been duplicated, one copy being situated immediately proximal to the inserted chromosome 1 segment (Figure $5)$.

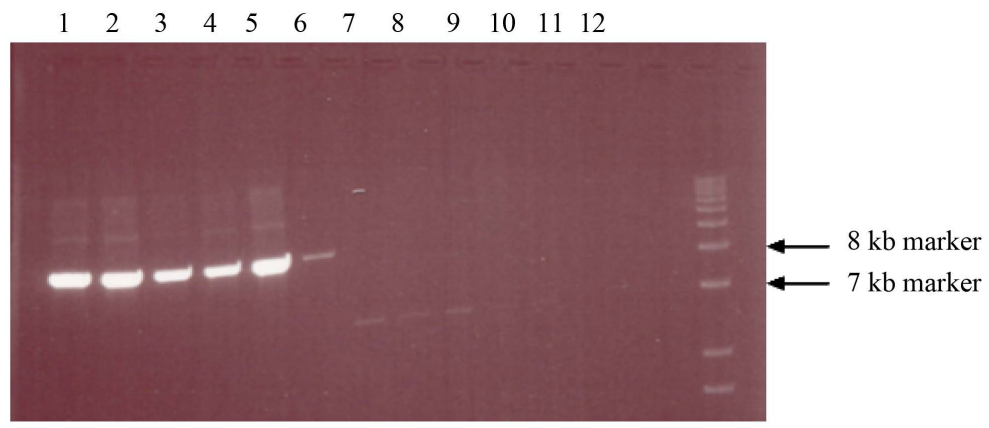

(a)

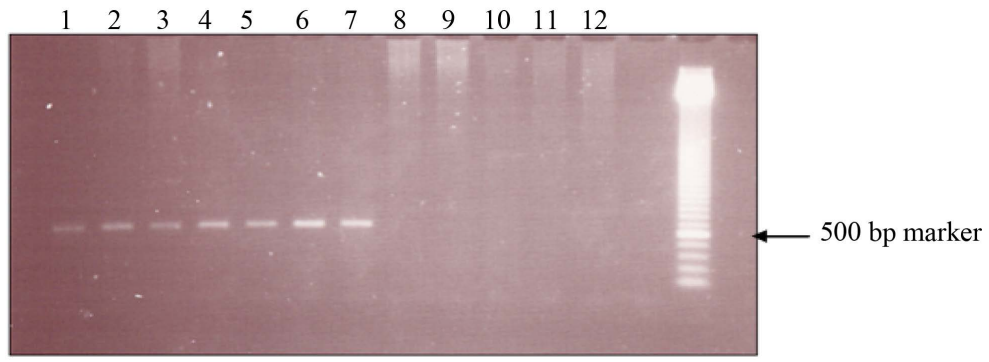

(b)

Figure 3. PCR amplification across the insertion breakpoint. (a) Gel electrophoresis of the products of PCR amplification across the putative site of insertion of chromosome 1 material into chromosome Xq27 using X specific primers. Tracks 1-6, DNA from unaffected males from the ptosis pedigree. Tracks 7-11, DNA from affected males from the ptosis pedigree. Track 12, control reaction containing no DNA. Size marker $=1 \mathrm{~kb}$ ladder. The primers amplify the expected $7.5 \mathrm{~kb}$ fragment from the DNA from unaffected males but not from DNA from affected males indicating the presence of the insertion in the affected males between the primers rendering the fragment too large to amplify by PCR. (b) Gel electrophoresis of the products of PCR amplification using a primer from chromosome 1 (bases 98,006,082-98,006,100) paired with a primer from chromosome X (bases 139,506,014-139,506,034). Tracks 1-7, DNA from affected individuals; tracks 8-12, DNA from unaffected individuals; track 13, no DNA control; track 14 size markers. A PCR product was generated from DNA from affected individuals but not with DNA from unaffected individuals, indicating that a breakpoint was within this fragment. 


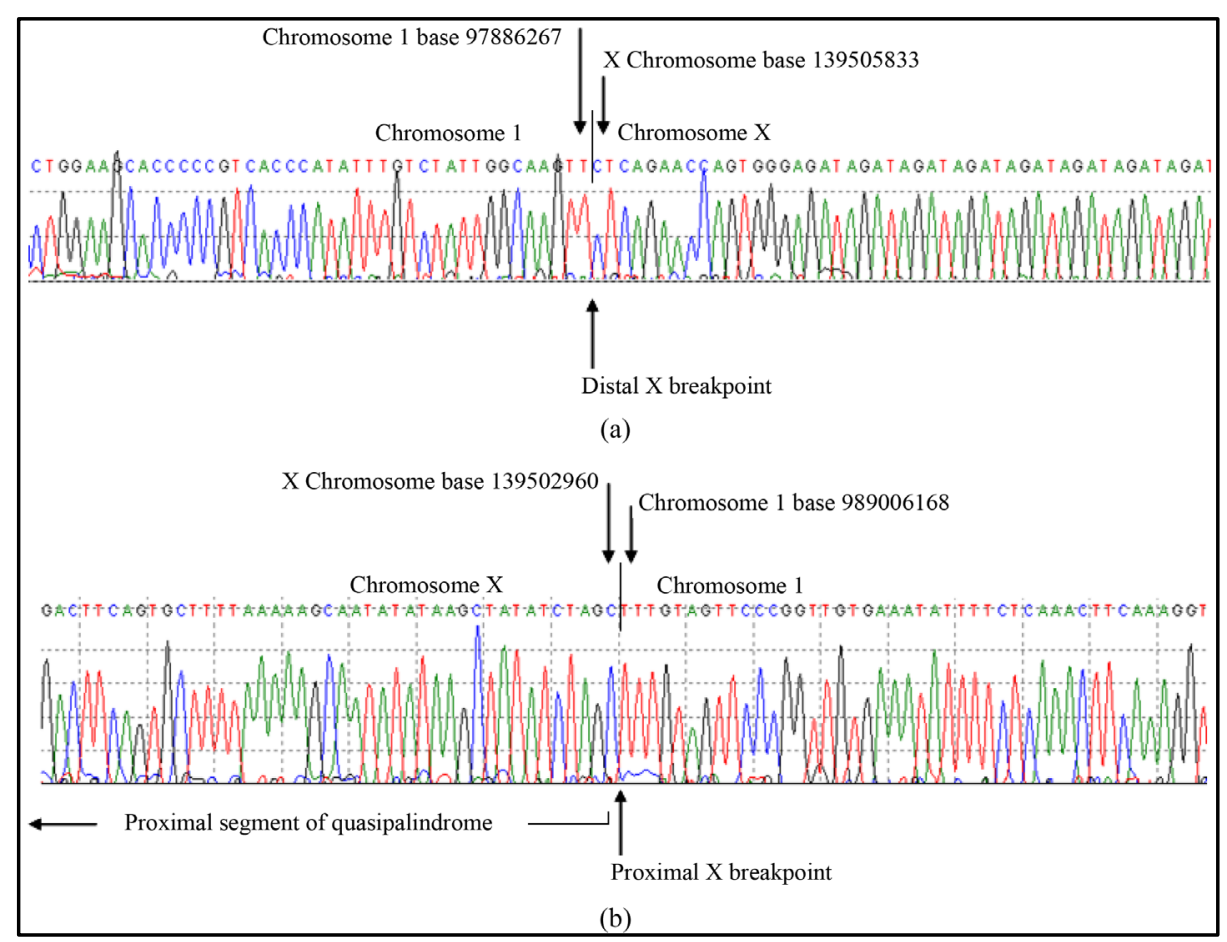

Figure 4. Breakpoint sequences. DNA sequence across the distal (a) and proximal (b) breakpoints of the chromosome 1 fragment insertion into chromosome Xq27.1.

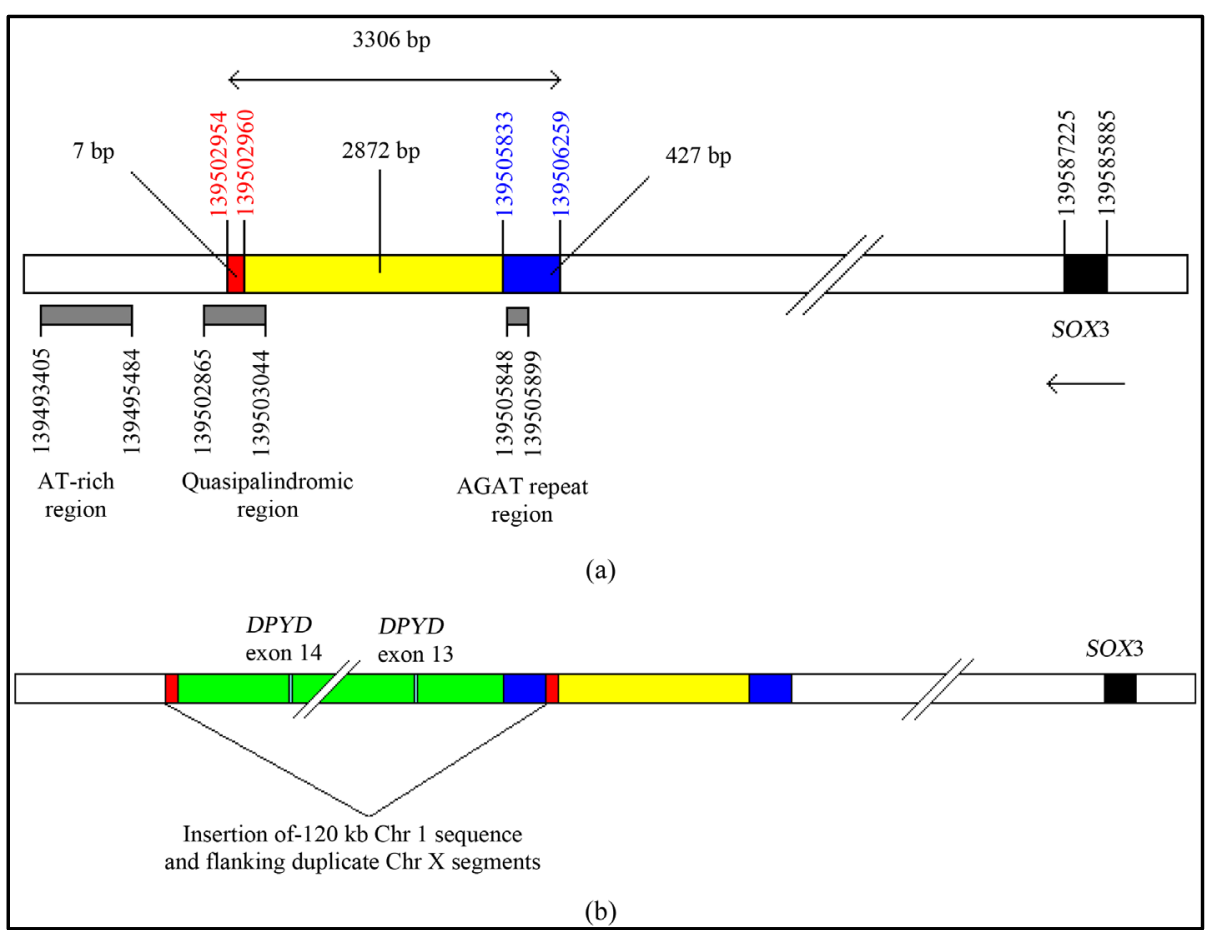

Figure 5. Structure of the X chromosome in the region of interest. (a) Normal X chromosome DNA sequence. (b) Sequence with chromosome 1 p21.3 material inserted and duplication of X chromosome sequences. A 427 base pair fragment (X: 139,505,833-139,506,259) has been duplicated, one copy of which is situated immediately distal to the inserted chromosome 1 segment. Also a 7 base pair sequence (X: 139,502,954-139,502,960) has been duplicated, one copy being situated immediately proximal to the inserted chromosome 1 segment. 
PCR amplification across the distal junction was carried out on DNA from all available family members. The chromosome 1 primer (bases 98,006,082-98,006,100) paired with the $\mathrm{X}$ chromosome primer (bases 139,506,014-139,506,034) gave an amplification product with all 19 available affected family members and two unaffected females with the at risk haplotype. No product was generated from 16 unaffected family members without the at risk haplotype (data not shown).

\subsection{Exome Sequencing}

No disease-causing mutations were identified within the $18 \mathrm{Mb}$ critical region of the $\mathrm{X}$ chromosome (or elsewhere in the genome). All detected changes were known variants or single nucleotide polymorphisms.

\section{Discussion}

We have identified an unbalanced insertional duplication of approximately $120 \mathrm{~kb}$ of the DPYD gene on chromosome 1p21.3 into Xq27.1, der (X) dir ins (X;1) (q27.1;p21.3), in a family with X linked dominant congenital bilateral isolated ptosis. The insertion is associated with a rearrangement of $\mathrm{X}$ chromosome sequences close to the site of insertion which includes a 427 base duplication and a 7 base duplication. The rearrangement segregates with the previously described familial at risk haplotype [1] which generated a LOD score of 5.89 and is present in all affected family members tested and in two unaffected females who also carry the at risk haplotype. One of these unaffected females has been previously shown to have severely skewed X inactivation [1] and also has an affected child with the rearrangement. The second unaffected female has a random pattern of $\mathrm{X}$ inactivation and it is unclear why she does not have the ptosis phenotype. Inactivation studies were performed on leukocyte DNA and it is possible that this may not reflect the pattern of $\mathrm{X}$ inactivation in other tissues.

Our strategy to use aCGH and FISH, followed by in silico analysis to identify likely regions on the $\mathrm{X}$ chromosome for the insertion, has been successful. The search for an AT-rich region identified such a locus close to the X chromosome breakpoint. This directed the targeting of PCR analysis and subsequent characterisation of the DNA base sequence of the breakpoints.

There is no direct evidence that this rearrangement causes X-linked bilateral congenital ptosis. The rearrangement segregates with $18 \mathrm{Mb}$ of chromosome $\mathrm{Xq}$ in all affected family members, so it is possible that a mutation elsewhere in this Xq fragment is causing the phenotype, although no evidence of a causative mutation was detected by exome sequencing. Such an insertion in this region of Xq is a rare event-we have only identified this one case in over 6000 samples analysed by array CGH in our laboratory, and we can only find three cases in the literature [4] [5]. We have found no other case with a partial duplication of DPYD.

There are a number of possible mechanisms whereby the rearrangement may cause the ptosis phenotype. It could be caused by 1 ) disruption of a gene on the $\mathrm{X}$ chromosome 2) trisomy for the inserted $D P Y D$ fragment 2) position effect, i.e. the disruption of regulatory sequences of a nearby gene by the DYPD insertion or $\mathrm{X}$ sequence rearrangement or 3) the generation of a hybrid transcript involving the translocated DPYD exons and the exons of a nearby gene.

The insertion does not disrupt a known gene on the $\mathrm{X}$ chromosome, the closest genes on either side being SOX3 (MIM 313430), a transcription factor which is $~ 80 \mathrm{~kb}$ distal to the insertion and CXorf66, a predicted type 1 membrane protein, which is $458 \mathrm{~kb}$ proximal to the insertion.

Trisomy for the inserted DPYD fragment seems an unlikely cause for the ptosis phenotype. There is a possibility that the translocated exons 13 and 14 of DPYD could be expressed from the X chromosome and compromise the function of the normal DPYD gene, resulting in dihydropyrimidine dehydrogenase deficiency (MIM 274270), but ptosis is not a feature of this condition. We can find no other cases of a duplication of part of $D P Y D$ in the literature.

Position effect is a possibility as the site of insertion of the DPYD fragment is only $80 \mathrm{~kb}$ downstream of the 3' end of SOX3, and there are numerous examples of position effect acting over this and much longer sequences of DNA [9]. The next closest genes to the insertion are much further away. CDR, a cerebellar degeneration related protein, is $359 \mathrm{~kb}$ upstream of the insertion and CXorf66, a predicted type 1 membrane protein, is $458 \mathrm{~kb}$ downstream. Although theoretically possible, position effect upon these genes has not been documented in the literature. However the action of position effect upon SOX3 has been proposed in a case with X-linked recessive hypoparathyroidism and an insertion of $340 \mathrm{~kb}$ of DNA from chromosome 2p25.3 into Xq27.1 and a 23 - $25 \mathrm{~kb}$ deletion of X chromosome sequence which did not include any genes but was $67 \mathrm{~kb}$ from the 3' end of SOX3 [4]. 
Similarly Zhu et al. [5] suggest position effect upon SOX3 in their description of two families with X-linked congenital hypertrichosis syndrome associated with insertions of $125 \mathrm{~kb}$ of chromosome $5 q 35.3$ and $300 \mathrm{~kb}$ of chromosome 4q31.2 respectively, both inserted at a site $\sim 80 \mathrm{~kb}$ downstream of SOX3 (discussed below). Congenital ptosis is not a feature of either of these syndromes and our ptosis pedigree shows no evidence of hypoparathyroidism or hypertrichosis.

Although there is evidence that position effect can influence SOX3 expression, there is no direct evidence that this may cause ptosis. Other phenotypes associated with $S O X 3$ mutation or altered expression include mental retardation with panhypopituitarism (OMIM 300123), 46,XX male sex reversal [10] and mental retardation with growth hormone deficiency [11]. Ptosis is not a feature of these phenotypes and conversely features of these phenotypes are not expressed in the ptosis family. However, the possibility of altered expression of SOX3 as the causative mechanism for the ptosis phenotype in this family is not excluded. SOX3 could influence innervation of the levator palpabrae superioris (LPS) muscle which raises the upper eyelid. The gene belongs to the SOXB1 subfamily of genes that are expressed throughout the developing central nervous system [12] and it has extensive homology with other transcription factors which are implicated in other ocular phenotypes, e.g. SOX2 [13] and FOXL2 [14] which are involved in the development of the eye and extraocular muscles.

The generation of a hybrid gene involving SOX3 and exons 13 and 14 of DPYD is a possibility. The two inserted DPYD exons are in the same orientation as the SOX3 gene and DPYD exon 13 is only 100 kb from the 3' end of SOX3. Such a hybrid would be likely to completely abrogate the functions of SOX3 and give a much more severe phenotype than ptosis, but if the hybrid transcript was present at a low level compared to the normal transcript it could have a lesser effect. $S O X 3$ is a single exon gene and the generation of such a hybrid transcript would require the use of a cryptic splice donor site within the gene, in frame with the inserted exons of the DPYD gene. Analysis using five splice site prediction programs (interactive-biosoftware.com/alamut) has identified a putative cryptic donor site within $S O X 3$ with a relatively high score for all 5 programs (core dinucleotide, SOX3 bases c.442 - 443, data not shown) which would remain in frame with the inserted DPYD exons, so this possibility is not excluded.

The nature of the rearrangement, as with many translocations [15], is more complex than a simple exchange. On the X chromosome, in the region of the insertion, segments of 427 bases and 7 bases have been duplicated (Figure 5). The DPYD gene fragment insertion was shown to be 119,902 bp in size $(1: 97,886,267-98,006,168)$ which includes the whole of exons 13 and 14 . The nature of any other rearrangement of chromosome 1p21.3 in the original recombination cannot be ascertained as all currently available members of the pedigree have the normal number of copies of DPYD on chromosome 1p21.3. With regard to the origin of the rearrangement, the DNA sequence of the $\mathrm{X}$ chromosome at and near the site of insertion shows some features that may confer instability on the area. The AT-rich region $7.5 \mathrm{~kb}$ proximal to the insertion site could be implicated in the rearrangement. Such regions are known to be associated with non-homologous recombination [4] [16] and are a common feature of fragile sites, components of normal chromosome structure prone to breakage [17]. Also, the duplicated region of the DPYD gene contains a known AT-rich fragile site [18]. Furthermore the $\mathrm{X}$ breakpoint lies within a quasipalindromic (imperfect inverted repeat) sequence of 180 bases which is the site of breakage in other documented cases with chromosome rearrangements in this region (discussed below). The nearby AT-rich region, with relatively low interstrand hydrogen bonding, may have allowed the generation of a single stranded region extending to the quasipalindromic sequence at the breakpoint. Instability at the quasipalindromic site would then be generated by intra-strand base pairing and strand breakage. An additional feature of the X chromosome rearrangement area is a sequence of 13 AGAT repeats which begins fifteen bases 3' to the distal breakpoint (Figure 4(a) and Figure 5). Such repeats are prone to replication slippage and therefore may also contribute to instability of the region.

Two cases with X-linked congenital hypertrichosis and insertional duplications into Xq27.1 described by Zhu et al. [5] both have almost identical X chromosome breakpoints to that in the ptosis family. Both cases have one of their breakpoints at X: 139,502,951, which is nine bases proximal to the ptosis pedigree breakpoint at X: 139,502,960. These breakpoints are within the 180 bp quasipalindromic sequence which appears to be a hotspot for genomic rearrangement. Zhu et al. [5] also describe the presence of deletions of this area with a breakpoint in the quasipalindrome in 9 out of 740 control individuals with no abnormal phenotype. Thus a breakpoint within this sequence is not sufficient to generate an abnormal phenotype. Also, the breakpoint alone does not define the nature of the abnormal phenotype as the hypertrichosis and ptosis phenotypes are clearly distinct even though the breakpoints are only 10 bases apart. The two Zhu et al. cases [5] have an insertion of different auto- 
somal sequences into Xq27.1, and one case was also shown to be deleted for $\mathrm{X}$ chromosome specific sequences. If modification of $S O X 3$ expression is causative of these phenotypes, it is not simply due to the presence of a breakpoint within the 180 bp quasipalindrome and must therefore be due to either the nature of the inserted sequence or the nature and position of the deleted or duplicated $\mathrm{X}$ chromosome sequences.

\section{Conclusions}

We have identified an insertion and duplication mutation in Xq27.1 cosegregating with X-linked congenital bilateral ptosis. The mutation is $\sim 80 \mathrm{~kb}$ from the 3' end of the SOX3 gene but we have no conclusive evidence to implicate $S O X 3$ with the ptosis phenotype. A comparison with other cases with almost the same X chromosome breakpoint suggests that the position of the breakpoint alone does not define the resultant phenotype, which must therefore be defined by the nature of the inserted sequence and/or the duplication or deletion of X chromosome material.

This case adds to the evidence that a 180 bp quasipalindromic sequence in Xq27.1 is a hot spot for genomic recombination in an area where complex rearrangements are associated with a number of different abnormal phenotypes, dependent upon the nature of the genetic material that is inserted at this point.

\section{Acknowledgements}

We thank the family members for participating in this study. The work was supported by the TFC Frost charitable trust, The Salisbury Medical Society, Salisbury Hospitals Foundation, The British Council for Prevention of Blindness, The Wessex Medical Trust and the Mason Medical Research Foundation.

\section{References}

[1] McMullan, T.F.W., Collins, A., Tyers, A.G. and Robinson, D.O. (2000) A Novel X Linked Truly Dominant Condition: X Linked Congenital Isolated Ptosis. American Journal of Human Genetics, 66, 1455-1460. http://dx.doi.org/10.1086/302860

[2] Engle, E.C., Castro, A.E., Macey, M.E., Knoll, J.H.M. and Beggs, A.H. (1997) A Gene for Isolated Congenital Ptosis Maps to a 3-cM Region within 1p32-p34. American Journal of Human Genetics, 60, 1150-1157.

[3] Møller, R.S., Jensen, L.R., Maas, S.M., Filmus, J., Capurro, M., Hansen, C., et al. (2014) X-Linked Congenital Ptosis and Associated Intellectual Disability, Short Stature, Microcephaly, Cleft Palate, Digital and Genital Abnormalities Define Novel Xq25q26 Duplication Syndrome. Human Genetics, 133, 625-638.

http://dx.doi.org/10.1007/s00439-013-1403-3

[4] Bowl, M.R., Nesbit, M.A., Harding, B., Levy, E., Jefferson, A., Volpi, E., et al. (2005) An Interstitial Deletion-Insertion Involving Chromosomes 2p25.3 and Xq27.1, near SOX3, Causes X-Linked Recessive Hypoparathyroidism. Journal of Clinical Investigations, 115, 2822-2831. http://dx.doi.org/10.1172/JCI24156

[5] Zhu, H., Shang, D., Sun, M., Choi, S., Liu, Q., Hao, J., et al. (2011) X-Linked Congenital Hypertrichosis Syndrome Is Associated with Interchromosomal Insertions Mediated by a Human-Specific Palindrome near SOX3. American Journal of Human Genetics, 88, 819-826. http://dx.doi.org/10.1016/j.ajhg.2011.05.004

[6] Schouten, J.P., McElgunn, C.J., Waaijer, R., Zwijnenburg, D., Diepvens, F. and Pals, G. (2002) Relative Quantification of 40 Nucleic Acid Sequences by Multiplex Ligation-Dependent Probe Amplification. Nucleic Acids Research, 30, e57. http://dx.doi.org/10.1093/nar/gnf056

[7] Li, H., Handsaker, B., Wysoker, A., Fennell, T., Ruan, J., Homer, N., et al. (2009) The Sequence Alignment/Map Format and SAM Tools. Bioinformatics, 25, 2078-2079. http://dx.doi.org/10.1093/bioinformatics/btp352

[8] Wang, K., Li, M. and Hakonarson, H. (2010) ANNOVAR: Functional Annotation of Genetic Variants from HighThroughput Sequencing Data. Nucleic Acids Research, 38, e164. http://dx.doi.org/10.1093/nar/gkq603

[9] Kleinjan, D.A. and van Heyningen, V. (2005) Long-Range Control of Gene Expression: Emerging Mechanisms and Disruption in Disease. American Journal of Human Genetics, 76, 8-32. http://dx.doi.org/10.1086/426833

[10] Sutton, E., Hughes, J., White, S., Sekido, R., Tan, J., Arboleda, V., et al. (2011) Identification of SOX3 as an XX Male Sex Reversal Gene in Mice and Humans. Journal of Clinical Investigations, 121, 328-341. http://dx.doi.org/10.1172/JCI42580

[11] Laumonnier, F., Ronce, N., Hamel, B.C., Thomas, P., Lespinasse, J., Raynaud, M., et al. (2002) Transcription Factor SOX3 Is Involved in X-Linked Mental Retardation with Growth Hormone Deficiency. American Journal of Human Genetics, 71, 1450-1455. http://dx.doi.org/10.1086/344661 
[12] Collignon, J., Sockanathan, S., Hacker, A., Cohen-Tannoudji, M., Norris, D., Rastan, S., et al. (1996) A Comparison of the Properties of Sox-3 with Sry and Two Related Genes, Sox-1 and Sox-2. Development, 122, 509-520.

[13] Fantes, J., Ragge, N.K., Lynch, S-A., McGill, N.I., Collin, J.R.O., Howard-Peebles, P.N., et al. (2003) Mutations in SOX2 Cause Anophthalmia. Nature Genetics, 33, 461-462. http://dx.doi.org/10.1038/ng1120

[14] Crisponi, L., Deiana, M., Loi, A., Chiappe, F., Uda, M., Amati, P., et al. (2001) The Putative Forkhead Transcription Factor FOXL2 Is Mutated in Blepharophimosis/Ptosis/Epicanthus Inversus Syndrome. Nature Genetics, 27, 159-166. http://dx.doi.org/10.1038/84781

[15] De Gregori, M., Ciccone, R., Magini, P., Pramparo, T., Gimelli, S., Messa, J., et al. (2007) Cryptic Deletions Are a Common Finding in "Balanced" Reciprocal and Complex Chromosome Rearrangements: A Study of 59 Patients. Journal of Medical Genetics, 44, 750-762. http://dx.doi.org/10.1136/jmg.2007.052787

[16] Edelmann, L., Spiteri, E., Koren, K., Pulijaal, V., Bialer, M.G., Shanske, A., et al. (2001) AT-Rich Palindromes Mediate the Constitutional t(11;22) Translocation. American Journal of Human Genetics, 68, 1-13. http://dx.doi.org/10.1086/316952

[17] Zlotorynski, E., Rahat, A., Skaug, J., Ben-Porat, N., Ozeri, E., Hershberg, R., et al. (2003) Molecular Basis for Expression of Common and Rare Fragile Sites. Molecular and Cellular Biology, 23, 7143-7151. http://dx.doi.org/10.1128/MCB.23.20.7143-7151.2003

[18] Hormozian, F., Schmitt, J.G., Sagulenko, E., Schwab, M. and Savelyeva, L. (2007) FRA1E Common Fragile Site Breaks Map within a 370 Kilobase Pair Region and Disrupt the Dihydropyrimidine Dehydrogenase Gene (DPYD). Cancer Letters, 246, 82-91. http://dx.doi.org/10.1016/j.canlet.2006.02.004 
Scientific Research Publishing (SCIRP) is one of the largest Open Access journal publishers. It is currently publishing more than 200 open access, online, peer-reviewed journals covering a wide range of academic disciplines. SCIRP serves the worldwide academic communities and contributes to the progress and application of science with its publication.

Other selected journals from SCIRP are listed as below. Submit your manuscript to us via either submit@scirp.org or Online Submission Portal.
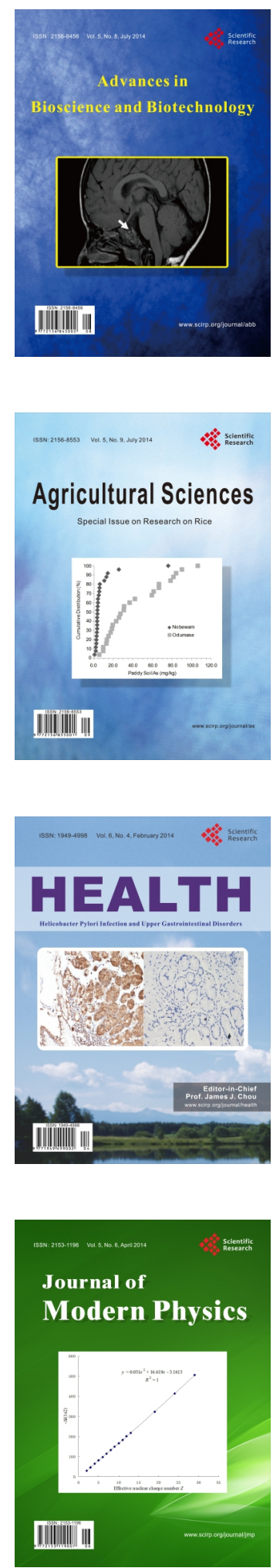
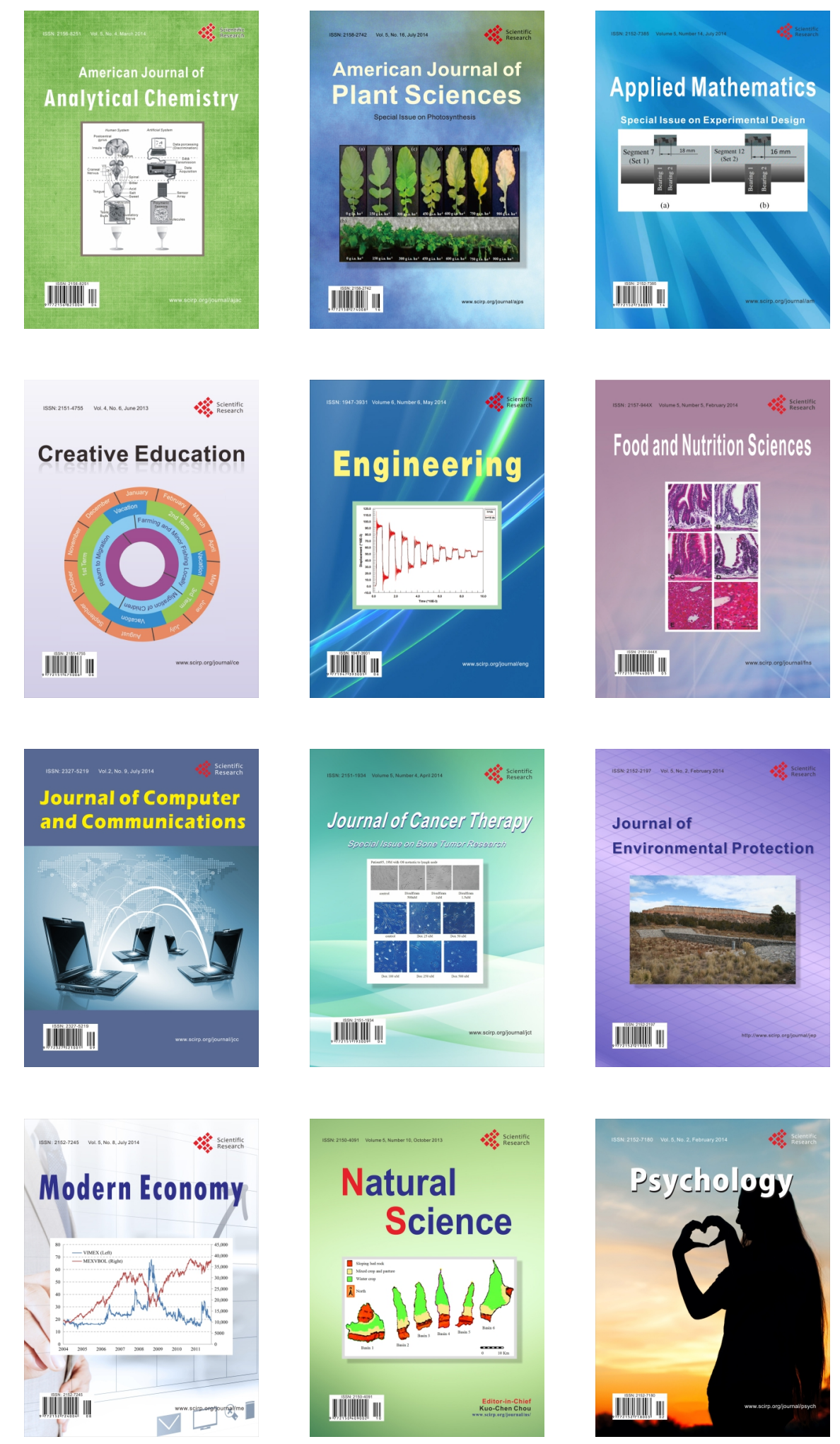